\section{Urología en el espacio y cambios genitourinarios en el ambiente de microgravedad}

Raúl Carrillo-Esper, ${ }^{1,4}$ Luis Daniel Carrillo-Córdova, ${ }^{2,3}$ Elba Luz Villena-López, ${ }^{4}$ Dulce María Carrillo-Córdova ${ }^{5,6}$

\section{Resumen}

EL objetivo actual de los vuelos tripulados en el espacio es la colonización de marte. Uno de los sistemas que se ve afectado con mayor frecuencia es el genitourinario, con afección en $10 \%$ de los astronautas, principalmente: litiasis, retención aguda de orina, infecciones de vías urinarias y alteraciones en la fertilidad. El objetivo de este estudio es revisar el efecto de los vuelos espaciales en el sistema genitourinario de los astronautas.

PALABRAS CLAVE: Litiasis; vuelos espaciales; fertilidad; infecciones urinarias.

Rev Mex Urol. 2018 March-April;78(2):162-167.

\section{Urology in space and genitourinary changes in the microgravity environment}

Raúl Carrillo-Esper, ${ }^{1,4}$ Luis Daniel Carrillo-Córdova, ${ }^{2,3}$ Elba Luz Villena-López, ${ }^{4}$ Dulce María Carrillo-Córdova ${ }^{5,6}$

\section{Abstract}

The current aim of manned spaceflight is the colonization of Mars. The genitourinary system is one of the most frequently affected in astronauts, occurring in $10 \%$ of them. The main pathologies are stone disease, acute urinary retention, urinary tract infections, and fertility alterations. The aim of the present study was to review the effect of space travel on the genitourinary systems of astronauts.

KEYWORDS: Stone disease; Spaceflight; Fertility; Urinary infections

\footnotetext{
1 Jefe de División CENIAQ, Instituto Nacional de Rehabilitación Luis Guillermo Ibarra Ibarra, Ciudad de México.

2 Departamento de Urología, Hospital General de México Dr. Eduardo Liceaga, Ciudad de México.

${ }^{3}$ Comité científico, Sociedad Mexicana de Medicina del Espacio y Microgravedad, Ciudad de México.

${ }^{4}$ Presidenta de la Sociedad Mexicana de Medicina del Espacio y Microgravedad, Ciudad de México.

${ }^{5}$ Medicina Interna, Hospital Ángeles del Pedregal, Ciudad de México.

${ }^{6}$ Medicina interna, Instituto Nacional de Ciencias Médicas y Nutrición Salvador Zubirán, Ciudad de México.
}

Recibido: noviembre 2017

Aceptado: marzo 2018

Correspondencia Luis Daniel Carrillo Córdova carrillocor@gmail.com

Este artículo debe citarse como Carrillo-Esper R, Carrillo-Córdova LD, Villena-López EL, Carrillo-Córdova DM. Urología en el espacio y cambios genitourinarios en el ambiente de microgravedad. Rev Mex Urol. 2018 marzo-abril;78(2): 162-167.

DOI: https://doi.org/10.24245/revmexurol.v78i2.1838 


\section{ANTECEDENTES}

El objetivo actual de los vuelos tripulados en el espacio es la colonización de marte. La distancia entre marte y la tierra es de 54.6 millones de kilómetros y con la tecnología existente esto llevaría, con el más prometedor de los cálculos, nueve meses de viaje espacial para llegar al planeta vecino.

Existen diversas alteraciones fisiológicas que afectan a los humanos durante las misiones al espacio. Uno de los sistemas afectados con mayor frecuencia es el genitourinario, que se ha reportado con alguna afección en 10\% de los astronautas que participaron en los vuelos o misiones espaciales entre 1981 y 1998, es decir, 89 vuelos y 508 astronautas. La medicina espacial ha tenido grandes avances, lo que ha hecho posible que se conozca a profundidad la adaptación fisiológica en microgravedad y los principales retos a los que se enfrentará el ser humano en los vuelos espaciales prolongados, en especial el viaje a Marte, proyecto programado para la década de 2030. Para esto se tiene la experiencia de estancias de más de un año del ser humano en un ambiente de microgravedad, de las que destacan las misiones de los cosmonautas rusos Valery Poliakov y Musa Maranov, y del astronauta norteameriano Scott Kelly en estancia prolongada, conjunta con Mikhail Kornienko. ${ }^{1}$

La medicina espacial se encarga de estudiar las modificaciones fisiológicas del ser humano en un ambiente de microgravedad, los mecanismos adaptativos y las enfermedades derivadas de la estancia en el espacio, su prevención y tratamiento. El pionero en esta rama de la medicina es el Dr. Ramiro Iglesias, destacado cardiólogo, especializado en medicina espacial, cuya visión del hombre y su adaptación a la migración al espacio se refleja en su libro: "La ruta hacia el hombre cósmico". México cuenta con una agencia espacial, que entre sus múltiples objetivos ha considerado a la medicina espacial como un proyecto prioritario, incluso se han sumado la Academia Nacional de Medicina, la Academia Mexicana de Cirugía, instituciones de educación superior e institutos de investigación. El trabajo conjunto de todos ellos se refleja en un documento titulado: "Medicina espacial", publicado por la Academia Nacional de Medicina de México y el Consejo Nacional de Ciencia y Tecnología, en el que se expone una visión integral de la medicina espacial y el plan estratégico del proyecto. ${ }^{2,3}$

El objetivo de este estudio es revisar puntualmente y poner a consideración de los interesados en esta importante área de la medicina espacial los efectos de los vuelos relacionados con el sistema genitourinario de los astronautas.

\section{Litiasis urinaria en los astronautas}

Los cálculos urinarios se han reportado en 1 astronauta durante el vuelo y en 11 astronautas después de su regreso del espacio. El episodio de litiasis en un vuelo se observó en un astronauta ruso, durante una misión de larga duración a bordo del Salyut. Este episodio estuvo cerca de arruinar la misión y ameritar una evacuación de urgencia, provocada por el dolor tan intenso que reportó el astronauta, debido a la obstrucción transitoria del uréter distal. Once astronautas de misiones a cargo de Estados Unidos reportaron 14 episodios de litiasis posterior a vuelos espaciales de dos semanas de duración. ${ }^{4,5}$

La exposición a la microgravedad genera alteraciones en los electrolitos séricos y urinarios. Los primeros estudios que señalaron resultados prometedores en astronautas con alteraciones hidroelectrolíticas se efectuaron en los vuelos que orbitaron la tierra: "Apollo". En éstos se evaluó el estado basal mineral óseo, los electrolitos séricos y urinarios de los astronautas durante el vuelo y posterior a éste. Entre los hallazgos reportados se encontró resorción ósea temprana e hipercalciuria. ${ }^{6}$ 
Se han realizado múltiples estudios para analizar la desmineralización ósea y los cambios en la dieta e ingesta de líquidos como causa de hipercalciuria, hiperuricosuria, hipocitraturia y $\mathrm{pH}$ ácido. Estas alteraciones se han reportado en los astronautas que tripulan la estación espacial internacional, como las causantes de la formación de cálculos durante la exposición a un ambiente de microgravedad. Del mismo modo se han desarrollado protocolos para disminuir la incidencia de litiasis en los astronautas mediante la prevención farmacológica y adecuación higiénico-dietética. $^{7-12}$

Los mecanismos asociados con riesgo elevado de originar cálculos en los astronautas son: reabsorción de calcio e hipercalciuria concomitante, hipocitraturia, deshidratación y falta de actividad física. ${ }^{13-15}$

Un factor que se encuentra en investigación es la función de las nanobacterias en el origen de litiasis en los seres humanos. Las nanobacterias fueron encontradas por primera vez en 1996 dentro de un meteorito marciano. Incluso se ha demostrado que muestran un crecimiento acelerado en condiciones de microgravedad y tienen capacidad única de desarrollar una capsula a base de cristales de calcio. Debido a este hallazgo, si se logra demostrar la relación entre las nanobacterias y la enfermedad litiásica en humanos, representaría una amenaza para las tripulaciones. ${ }^{16-17}$

Se ha encontrado que la exposición a un estado de microgravedad provoca pérdida mineral ósea acelerada; sin embargo, la causa exacta del mecanismo aún se desconoce. Algunos estudios experimentales in vitro señalan que ante la ausencia de estímulo por la fuerza de gravedad, la reabsorción ósea se origina, principalmente, por la hiperactividad de los osteoclastos y disminución de la función de los osteoblastos (Figura 1). Como resultado los astronautas se someten a actividad física extenuante, mediante un dispositivo Ilamado Advanced Resistive Exercise
Device (ARED), que permite realizar ejercicio anaeróbico con resistencias durante las misiones y se ha relacionado con disminución de la pérdida mineral ósea, ganancia en masa muscular y disminución de tejido adiposo. ${ }^{18}$

También se ha evaluado si la prescripción de fármacos puede disminuir la pérdida mineral ósea (bisfosfonatos mediante la apoptosis de los osteoclastos) y provocar hipercalciuria. Un trabajo conjunto entre Japón y EUA, efectuado en astronautas a bordo de la estación espacial internacional, quienes recibieron $70 \mathrm{mg} / \mathrm{semanal}$ de alendronato antes y durante su vuelo, además del dispositivo ARED, reportó una disminución significativa de pérdida mineral ósea y calcio urinario. $^{18}$

Además de estos fármacos se han evaluado otros medicamentos que disminuyen la incidencia de cálculos renales en los astronautas. Un estudio doble ciego, con placebo, analizó el efecto de la administración de $20 \mathrm{mEq}$ de citrato de potasio en 18 astronautas a bordo de la estación espacial internacional. Los astronautas que recibieron citrato mostraron un aumento en el $\mathrm{pH}$ urinario y menor excreción urinaria de calcio, que resultó en menor saturación de ácido úrico y oxalato de calcio, además de menor riesgo de padecer litiasis. ${ }^{19-20}$ El Cuadro 1 muestra las causas y estrategias de prevención de litiasis en astronautas.

Cuadro 1. Causas de litiasis en astronautas y estrategias de prevención

\begin{tabular}{l|l}
\hline Etiología & Prevención específica \\
\hline $\begin{array}{l}\text { Falta de actividad física } \\
\text { secundaria a un ambiente de } \\
\text { microgravedad }\end{array}$ & $\begin{array}{l}\text { Advanced Resistive } \\
\text { Exercise Device (ARED) }\end{array}$ \\
$\begin{array}{l}\text { Hiperactividad de osteo- } \\
\text { clastos e hipoactividad de } \\
\text { osteoblastos }\end{array}$ & Bisfosfonatos \\
\hline $\begin{array}{l}\text { Hipocitraturia e hipercal- } \\
\text { ciuria }\end{array}$ & Citrato de potasio \\
\hline $\begin{array}{l}\text { Nanobacterias } \\
\begin{array}{l}\text { Estasis urinaria e infecciones } \\
\text { de repetición }\end{array}\end{array}$ & $\begin{array}{l}\text { Antibióticos profilácticos } \\
\text { de orina }\end{array}$ \\
\hline
\end{tabular}



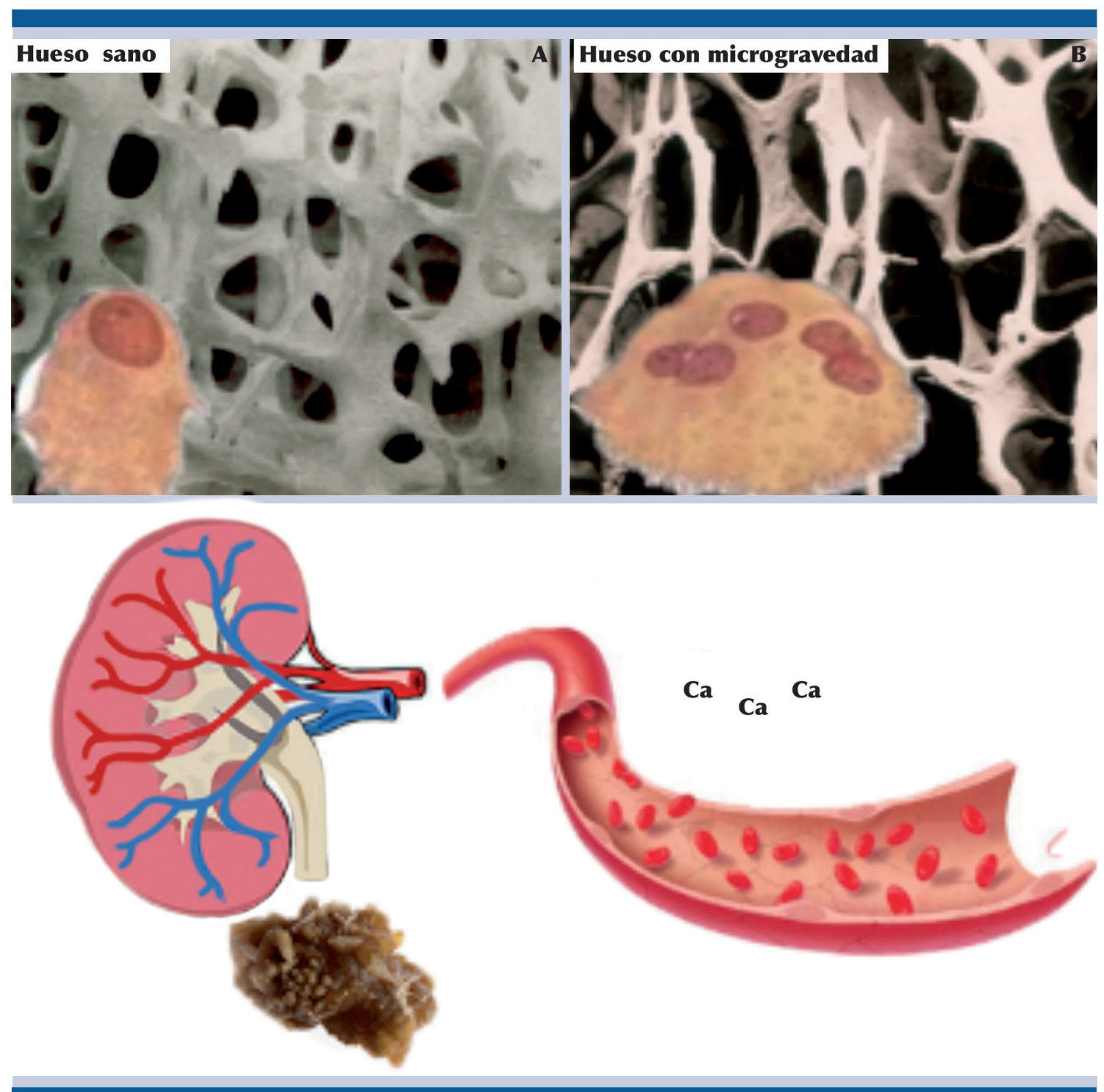

Figura 1. Proceso de formación de cálculos renales en un entorno de microgravedad. La actividad acelerada de los osteoclastos y la hipoactividad de los osteoblastos provoca desmineralización ósea acompañada de salida de calcio del hueso al torrente sanguíneo, evento que condiciona hipercalcemia con hipercalciuria y subsecuentemente la formación de orina sobresaturada de calcio que forma los cálculos. A: osteoblasto, B: osteoclasto.

\section{Infecciones de la vía urinaria}

En cuanto a infecciones de la vía urinaria se han reportado múltiples cuadros en astronautas. Entre los factores desencadenantes se ha sugerido el estrés emocional y físico al que se someten los tripulantes de las misiones espaciales, además de la deshidratación y problemas de higiene. 
Incluso se ha señalado que los sistemas de colecta de orina y el acceso restringido para orinar repercuten en estos casos. Un estudio reportó un caso de sepsis a bordo del vuelo espacial Ruso Salyut, secundario a prostatitis, que requirió la extracción medica de urgencia del astronauta. ${ }^{21-22}$

El diagnóstico de infección de vías urinarias en los vuelo espaciales se ha establecido mediante bandas (tiras reactivas) de análisis químico de orina (EUA) y con un analizador de orina dentro de la nave (Rusia). Los síntomas y la expresión de esterasa leucocitaria o nitritos en la banda han comprobado el diagnostico de infección de vías urinarias y el tratamiento consistió en antibióticos orales encontrados en el dispositivo o paquete médico ambulatorio. Las medidas sugeridas para disminuir la incidencia de infección de vías urinarias consiste en adecuada higiene perineal, utilizar un sistema de recolección de orina limpio, adecuada hidratación y micción programada. $^{22}$

\section{Retención aguda de orina}

Se han reportado cuatro casos de retención de orina durante los vuelos espaciales, que incluso requirieron cateterización vesical para curarse. El origen de estos cuadros fue multifactorial: consumo de fármacos anticolinérgicos y antieméticos (prescritos para el mareo), retraso en la micción y alteraciones en la sensación miccional. Una preocupación de los vuelos de mayor duración (misión a marte) es la posible retención aguda de orina secundaria a crecimiento prostático o la coexistencia de un cálculo uretral. Para prevenir estas complicaciones debe realizarse la selección estricta de la tripulación, establecer programas de prevención asociados con la formación de cálculos y el origen de infecciones de vías urinarias.
Fertilidad en el espacio

Puesto que el objetivo de la exploración espacial es la colonización de marte, el estudio de fertilidad humana es un punto angular en la evaluación del hombre en el espacio. Las preguntas que nos hemos realizado incluyen: ¿cuál es la función de la microgravedad en la espermatogénesis y embriogénesis? ¿cuál es el efecto de la radiación en el desarrollo embrionario y el estrés físico y mental al que están sometidos los astronautas y su relación con la desregulación hormonal?. En condiciones de microgravedad los fluidos tienden a migrar hacia la cavidad torácica, el cuello y la cabeza, y con ello provocar cierto grado de hipokalemia. Se ha postulado que esta redistribución de líquidos puede desencadenar la formación de embriones más pequeños por la falta de nutrientes..$^{23,24}$

Los primeros en estudiar la posibilidad de embarazos en vuelos espaciales fueron los rusos, a bordo del Cosmos 1129, en cuya misión de 18.5 días demostraron la fertilidad en ratas que regresaron del vuelo espacial y embarazos logrados en el espacio; sin embargo, no se reportó ningún nacimiento con feto vivo. ${ }^{21}$ Algunos estudios sugieren alteraciones en los espermatozoides en un ambiente de microgravedad, por ejemplo: cambios en la velocidad y morfología del flagelo, retraso en la respuesta a los factores quimiotácticos del óvulo y menor fertilidad. ${ }^{25-26}$

Los datos actuales señalan que la fertilidad es la misma que en la tierra después de una semana de permanecer en el entorno de microgravedad; entre los hallazgos más importantes se han reportado: 56 embarazos posteriores a vuelos espaciales, 11 embarazos en el año posterior al vuelo espacial, 17 partos en astronautas que viajaron embarazadas, 11 abortos, 2 nacimientos con enfermedades genéticas crónicas y 43 niños sanos. ${ }^{26-27}$ 


\section{CONCLUSIONES}

El efecto de los vuelos espaciales prolongados en el sistema genitourinario es bien conocido y afectan a $10 \%$ de los astronautas. Los estudios llevados a cabo en esta área han ayudado a entender los cambios fisiopatológicos a los que se exponen los astronautas, desarrollar medidas preventivas para disminuir la incidencia de enfermedades y, sobre todo, dar un paso más hacia nuestro objetivo final en la carrera espacial: la colonización de marte.

\section{REFERENCIAS}

1. The human body in the space [en línea]. Dirección URL: <https://www.nasa./hrp/bodyinspace >.

2. Iglesias Leal, Ramiro. La Ruta Hacia el Hombre Cósmico". Ed. Limusa. 2AD

3. Carrillo Esper, R. Medicina Espacial. Documento de Postura. Academia Nacional de Medicina de México [en línea]. Dirección URL: <https://www.anmm.org.mx/publicaciones/ CAmvANM150/Medicina_Espacial.pdf $\geq$.

4. Pietrzyk RA, Jones JA, Sams CF, Whitson PA. Renal stone formation among astronauts. Aviat Space Environ Med 2007;78:9-13.

5. Pietryzk R, Whitson P, Jones J, Sams C. Overview of renal stones and space flight. Bulletin of the 14th International Academy of Astronautics-Humans in Space Symposium: Living in Space: Scientific, Medical, and Cultural Implications, Banff, Alberta, Canada, May 22/2003.

6. Johnston RS, Dietlein LF, Berry CA, et al. Biomedical results of Apollo, Scientific and Technical Information Office, NASA Headquarters, Washington, D.C., 1975.

7. Whitson PA, Pietrzyk RA, Pak CY. Renal stone risk assessment during Space Shuttle flights. J Urol 1997;158:2305-2310.

8. Whitson PA, Pietrzyk RA, Morukov BV, et al. The risk of renal stone formation during and after long duration space flight. Nephron 2001;89:264-270.

9. Hoyer JR, Pietrzyk RA, Liu H, et al. Effects of microgravity on urinary osteopontin. J. Am. Soc. Nephrol. 1999;10(Suppl: 14):S389-93.

10. Smith SM, Zwart SR, Heer M, et al. Men and women in space: bone loss and kidney stone risk after long-duration spaceflight. J Bone Miner Res 2014;29:1639-1645.

11. Kumei $Y$, Shimokawa $H$, Katano $H$, et al. Microgravity induces prostaglandin E2 and interleukin-6 production in normal rat osteoblasts: role in bone demineralization. J Biotechnol 1996;47:313-324.
12. Whitson PA, Pietrzyk RA, Pak CY, et al. Alterations in renal stone risk factors after space flight. J Urol 1993;150:803-807.

13. Jones JA. Association of calcifying nanoparticles within serum and prostates of patients with prostatic inflammation and hyperplasia: a preliminary analysis. Society of Inflammation and Infection in Urology Session at the American Urological Assn. Annual Meeting, San Antonio, TX 2005 May.

14. Kajander OE, Ciftcioglu N, Katya A, Garcia-Cuerpo E. Characteristics of nanobacteria and their possible role in stone formation. Urol Res 2003;31:47-54.

15. Smith SM, Heer MA, Shackelford LC et al. Benefits for bone from resistance exercise and nutrition in long-duration spaceflight: Evidence from biochemistry and densitometry. J Bone Miner Res 2012;27:1896-1906.

16. Leblanc $\mathrm{A}$, Matsumoto $\mathrm{T}$, Jones, J et al. Bisphosphonates as a supplement to exercise to protect bone during longduration spaceflight. Osteoporos Int 2013;24:2105-2114.

17. Zerwekh JE, Odvina CV, Wuermser LA, et al. Reduction of renal stone risk by potassium-magnesium citrate during 5 weeks of bed rest. J Urol 2007;177:2179-2184.

18. Whitson PA, Pietrzyk RA, Jones JA, et al. Effect of potassium citrate therapy on the risk of renal stone formation during spaceflight. J Urol 2009;182:2490-2496.

19. Pietrzyk RA, Whitson P, Jones J, Sams C. Renal stone risk during space flight assessment and countermeasure validation. USRA/DSLS Bioinvestigator's Workshop Abstract Volume. Annual Meeting. Galveston, TX, 2005 January 10-12.

20. Jones JA, Whitson P. Genitourinary issues in space medicine. In: Barratt M (ed). Clinical Space Medicine 2005.

21. Jones JA, Jennings R, Pietryzk R, Ciftcioglu N, Stepaniak P. Genitourinary issues during spaceflight: a review. Int J Impot Res 2005;17(Suppl 1):S64-7.

22. Stepaniak PC, Ramchandani SR, Jones JA. Acute urinary retention among astronauts. Aviat Space Environ Med 2007;78:5-8

23. Warren MP. Effects of space travel on reproduction. Obstet Gynecol Surv 1989;44:85-88

24. Ouza K, Hogan R, Ballard R. Life into Space: Space Life Sciences Experiments at Ames Research Center 1965-1990. NASA RP-1372, Washington,DC, 1995.

25. Tash JS, Kim S, Schuber M, et al. Fertilization of sea urchin eggs and sperm motility are negatively impacted under low hypergravitational forces significant to space flight. Biol Reprod 2001;65:1224-1231.

26. Tash JS, Bracho GE. Microgravity alters protein phosphorylation changes during initiation of sea urchin sperm motility. FASEB J 1999;13(Suppl 1):S43-54.

27. Ronca AE, Baker ES, Bavendam GT, Beck KD, Miller MV, et al. Effects of Sex and Gender on Adaptations to Space: Reprodctive Health. J Womens Health 2014;23:967-974. 